\title{
Evaluation of the depth of sedation in an intensive care unit based on the photo motor reflex variations measured by video pupillometry
}

Ouri Rouche ${ }^{1 *}$, Aurore Wolak-Thierry², Quentin Destoop ${ }^{1}$, Lucas Milloncourt ${ }^{1}$, Thierry Floch ${ }^{1}$, Pascal Raclot ${ }^{1}$, Damien Jolly ${ }^{2}$ and Joël Cousson ${ }^{1}$

\begin{abstract}
Background: Evaluating depth of sedation in the intensive care unit (ICU) is crucial for the management of mechanically ventilated patients but can be challenging in some situations. Because the depth of hypnosis is correlated with the decrease in photomotor reflex (PMR), we suggest using pupillometric video as an automated, noninvasive, simple, and reproducible technique to evaluate the depth of sedation in ICU patients. We compare the effectiveness of this procedure to the bispectral index (BIS).

Methods: Thirty-one patients requiring sedation and ventilation were included in this monocentric, observational study. The posology of hypnotics and morphinics were based on the Richmond Agitation and Sedation Scale (RASS). PMR was measured by the Neurolight ${ }^{\circledR}$ (IDMED) system and BIS value by BIS Vista ${ }^{\circledR}$ (Anandic Medical Systems). RASS, PMR, and BIS were measured three times daily in all patients. Data acquired by pupillometric video included variation in pupillary diameter $(\triangle P D)$, latency time $(L T)$, and maximal speed of pupillary constriction (Vmax). These parameters were analyzed after having classified BIS values in three groups ( $<40$ heavy sedation; $40 \leq$ BIS $\leq$ 60 acceptable sedation; $>60$ light sedation). Exclusion criteria were neurological or ophthalmologic pathologies that could interfere with PMR.
\end{abstract}

Results: There was a significant difference in Vmax and $\triangle P D$ between the BIS $<40$ group and $40 \leq B I S \leq 60$ groups ( $p<0.0001$ for each) and between the BIS $<40$ and BIS $>60$ groups ( $p<0.0001$ for each). There were no significant differences in Vmax and $\triangle \mathrm{PD}$ between the $40 \leq \mathrm{BIS} \leq 60$ and BIS $>60$ groups. There was no correlation between any of the BIS groups and LT.

Conclusions: Vmax and $\triangle P D$ seem to be relevant criteria compared with the BIS and the RASS. Pupillometric video monitoring of depth of sedation could be beneficial in ICU patients, especially for those under myorelaxant drugs, where no clinical evaluation of sedation is possible.

Keywords: Sedation, Photomotor reflex, Video pupillometry, Bispectral index

\section{Background}

Large numbers of patients admitted to the intensive care unit (ICU) are sedated when mechanically ventilated [1]. Evaluating the depth of sedation is a crucial element in the management of these patients. Indeed, when sedation is too deep, it can lead to hemodynamic instability, and when too light, it can cause ventilatory difficulties,

\footnotetext{
* Correspondence: ourilico@hotmail.com

${ }^{1}$ Service de Réanimation Polyvalente Hôpital Robert Debré, CHU 51092 Reims Cedex, France

Full list of author information is available at the end of the article
}

accidental extubation, or memorization phenomena. Assessing depth of sedation may be difficult in some situations. For example, in patients with acute respiratory distress syndrome (ARDS), ventilation often is difficult and may require myorelaxant drugs, which make it impossible to evaluate clinically the depth of sedation. In these cases, current practice suggests shortly interrupting the administration of these drugs on a daily basis to evaluate correctly the sedation level of the patient.

\section{里}

C 2013 Rouche et al.; licensee Springer. This is an Open Access article distributed under the terms of the Creative Commons Attribution License (http://creativecommons.org/licenses/by/2.0), which permits unrestricted use, distribution, and reproduction in any medium, provided the original work is properly cited. 
To optimize the quality of sedation, several review articles and guidelines have been published that outline recommended management [2-4]. Because the depth of hypnosis is known to be correlated with a decrease in photomotor reflex (PMR) [5-10], it could be beneficial to develop an automated, noninvasive, simple, and reproducible technique to evaluate efficiently the depth of sedation in an ICU based on the pupillary reflex.

Depth of sedation is not commonly monitored by the bispectral index (BIS) in the ICU setting; its use is controversial. Several studies have attempted to validate this device compared with commonly used sedation scales. BIS values suffer from a wide dispersion of values compared with predefined clinical scale scores. The main challenge is the overestimation of BIS values due to electromyographic activity [11]. In deeply sedated patients, values remain widely dispersed but are correlated to clinical sedation scale values (e.g., RASS -5).

The objective of this monocentric observational study was to evaluate the effectiveness of pupillometric video using the Neurolight ${ }^{\circledR}$ (IDmed ${ }^{\mathrm{TM}}$ ) system compared with the Richmond Agitation and Sedation Scale (RASS) [12-14] and the bispectral index (BIS).

\section{Methods}

\section{Study population}

Patients hospitalized in the ICU of Reims ChampagneArdennes University Hospital between 1 June and 31 August 2011 were eligible for inclusion in this study. The ICU has a capacity of 15 beds with a nurse to patient ratio of 1:3. All ICU patients who required heavy sedation to be adapted optimally to mechanical ventilation were included in the study. The study protocol was approved by the ethics committee of our institution. Exclusion criteria included all intrinsic or extrinsic patient factors that could interfere with PMR values, namely: i) use of any of the following drugs: clonidine, tramadol, droperidol, metoclopramide, ketamine, adrenalin, or calcium channel blockers $[8,15,16]$; ii) neurological pathologies (severe head trauma, subarachnoid hemorrhage, stroke, intracerebral hemorrhage, or multiple sclerosis); and iii) ophthalmological pathologies (cataract, conjunctivitis, or high myopia).

\section{Data recorded}

The doses of midazolam and sufentanil were based on the RASS, with a target score between RASS -4 and -5 . These drugs, which are commonly used in the ICU, do not alter the PMR $[6,10]$.

PMR was measured with a 320 Lux flash of light using the Neurolight ${ }^{\circledR}$ (IDmed, Marseille, France). Three measurements per day were taken for a 48-hour period. BIS values also were recorded (BIS Vista ${ }^{\circledR}$, Anandic
Medical Systems, Feuerthalen, Switzerland). The data recorded by the pupillometric video comprised: variation of pupillary diameter $(\triangle \mathrm{PD})$, latency time $(\mathrm{LT})$, and maximal speed of pupillary constriction (Vmax). These measurements were analyzed after classification of BIS values into three groups ( $<40$ heavy sedation; $40 \leq$ BIS $\leq 60$ acceptable sedation; $>60$ light sedation) [17]. All assessments of PMR, BIS, and RASS followed a standardized protocol for the study. Each patient had three evaluations per day, at predefined hours and under equivalent lighting conditions. The same physician performed all measurements (O.R.).

\section{Number of measures needed Statistical analysis}

Quantitative variables are presented as mean \pm standard deviation (SD) and qualitative variables as number and percentage. After having verified that the distribution was normal, we conducted repeated measures analysis of variance (ANOVA) for the variables of interest (i.e., $\triangle \mathrm{PD}$, LT, Vmax). If repeated measures ANOVA was significant, we considered each measure independently and performed one-way ANOVA adjusted for the subject, day, and measure to compare pupillometry parameters between the three BIS groups. When a significant difference was found, two by two comparisons between the three groups of BIS was performed using Bonferroni tests.

For all analysis, $p<0.05$ was considered statistically significant. Analyses were performed using SAS version 9.3 (SAS Institute Inc., Cary, NC).

\section{Results}

A total of 167 measures of PMR and BIS were conducted on 31 patients. The patient characteristics are summarized in Table 1. The medication taken by the patients during the study period is detailed in Table 2 . There were no significant differences between groups in LT. Repeated measures ANOVA showed significant differences for Vmax and $\triangle \mathrm{PD}(p<0.0001)$. Subsequent two by two comparison using Bonferroni tests revealed significant differences between the groups BIS $<40$ and $40 \leq \mathrm{BIS} \leq 60$, and between the BIS $<40$ and BIS $>60$ groups for $\operatorname{Vmax}$ and $\triangle \mathrm{PD}(p<0.0001$ for all comparisons; Table 3).

\section{Discussion}

Our results show that depth of sedation seems to be efficiently evaluated by the PMR measured by the Neurolight ${ }^{\circledR}$. The PMR was correlated to the RASS objectives. Compared with the BIS groups, a significant difference was found between the groups BIS $<40$ and $40 \leq \mathrm{BIS} \leq 60$, and between the BIS $<40$ and BIS $>60$. We could not show a difference between the groups $40 \leq$ 
Table 1 Baseline characteristics of the study population

\begin{tabular}{lc}
\hline Variable & All patients $(\mathbf{n}=\mathbf{3 1})$ \\
\hline Age $(\mathrm{yr})$ & $57 \pm 14$ \\
Weight $(\mathrm{kg})$ & $81.7 \pm 18.4$ \\
Height $(\mathrm{cm})$ & $166 \pm 32$ \\
$\mathrm{SAPS} \|$ & $56.3 \pm 15.8$ \\
$\mathrm{PaO}_{2} / \mathrm{FiO}_{2}(\mathrm{mmHg})$ & $194 \pm 114$ \\
$\mathrm{RASS}_{\text {score }}$ & $-4.45 \pm 0.71$ \\
Male sex & $23(74.2)$ \\
Myorelaxant drugs & $20(64.5)$ \\
Death in ICU & $20(64.5)$ \\
Indications for ICU entry & \\
Septic shock & $20(64.5)$ \\
Cardio circulatory arrest & $8(25.8)$ \\
Status epilepticus & $1(3.23)$ \\
Acute severe asthma & $1(3.23)$ \\
Coma & $1(3.23)$ \\
\hline
\end{tabular}

$\mathrm{Kg}$, kilograms; cm, centimeters; SAPS II, Simplified Acute Physiology Score; $\mathrm{PaO}_{2}$, partial pressure of oxygen in arterial blood; $\mathrm{FiO}_{2}$, fraction of inspired oxygen; ICU, intensive care unit.

Table 2 Average doses used for sedation and medications taken by the study population during the study period

\begin{tabular}{|c|c|c|}
\hline Sedation & $\mathbf{N}$ & Mean (SD) \\
\hline Pentothal (mg/h) & 1 & 350.00 \\
\hline Sufentanil (gamma/h) & 31 & $20.00(5.24)$ \\
\hline Midazolam (mg/h) & 31 & $11.61(4.72)$ \\
\hline Medication & $\mathrm{N}$ & $\%$ \\
\hline Norepinephrine & 26 & 83.87 \\
\hline Enoxaparin & 14 & 45.16 \\
\hline Gentamicin & 12 & 38.71 \\
\hline Ceftriaxone & 10 & 32.26 \\
\hline Aspirin & 10 & 32.26 \\
\hline Atorvastatin & 8 & 25.81 \\
\hline Fondaparinux & 6 & 19.35 \\
\hline Amiodarone & 6 & 19.35 \\
\hline Dobutamine & 6 & 19.35 \\
\hline Ticarcillin/clavulanic acid & 5 & 16.13 \\
\hline Clopidogrel & 5 & 16.13 \\
\hline Furosemide & 4 & 12.90 \\
\hline Piperacillin & 3 & 9.68 \\
\hline Metronidazole & 2 & 6.45 \\
\hline Ceftazidime & 1 & 3.23 \\
\hline Levofloxacin & 1 & 3.23 \\
\hline Imipenem - cilastatin sodium & 1 & 3.23 \\
\hline Vancomycin & 1 & 3.23 \\
\hline
\end{tabular}

$\mathrm{SD}$, standard deviation.
Table 3 Adjusted means of pupillometric measures (Vmax, LT, and $\triangle P D$ ) for each BIS group

\begin{tabular}{lccc}
\hline \multicolumn{3}{c}{ BIS } \\
\hline Pupillometric & $\mathrm{BIS}<40$ & $40 \leq \mathrm{BIS} \leq 60$ & $\mathrm{BIS}>60$ \\
Measures & $(\mathrm{N}=68)$ & $(\mathrm{N}=62)$ & $(\mathrm{N}=37)$ \\
$V \max (\mathrm{mm} / \mathrm{s})$ & 1.14 & 1.35 & 1.40 \\
$\mathrm{LT}(\mathrm{ms})$ & 259.25 & 237.92 & 228.00 \\
$\Delta \mathrm{PD}(\mathrm{mm})$ & 0.3 & 0.4 & 0.43 \\
\hline$p<0.0001$ for $V \max$ and $\Delta \mathrm{PD}$ between $\mathrm{BIS}<40$ and $40 \leq \mathrm{BIS} \leq 60$ groups. \\
$p<0.0001$ for $\operatorname{mmax}$ and $\Delta \mathrm{PD}$ between $\mathrm{BIS}<40$ and BIS $>60$ groups.
\end{tabular}

BIS $\leq 60$ and BIS $>60$, probably because of the limitations of the BIS use in ICU and perhaps because we did not have enough measurements.

Other studies showed similar interest in the PMR for the evaluation of the sedation. These studies did not concern ICU patients; however, they all showed that the LT variable was probably of no interest in the evaluation of sedation $[18,19]$.

This device appears to be of utility in the ICU, where patients can often be treated by myorelaxant drugs (muscle-blocking drugs do not alter the PMR [20]), because no clinical scale can be used in this situation. The utility of the BIS in the ICU remains to be defined [21], but it is undoubtedly still useful when clinical scales are ineffective, especially when muscular relaxant drugs are used [21-28]. Actually, there is no "gold standard" for evaluating the sedation status in patients under myorelaxant drugs.

Current guidelines recommend interruption of myorelaxant infusion and clinical evaluation of the sedation level when the muscle blockade has been reversed. However, these guidelines can sometimes be difficult to implement, due to the very unstable status of this category of critically ill patients.

The Neurolight ${ }^{\circledR}$ manufacturer (IDmed ${ }^{\mathrm{TM}}$ ) gives an indication of the pupil reactivity (very good, good, week, null) using all three variables measured by the device. To the best of our knowledge, no thresholds have been defined for pupillometry through a large-scale, randomized, controlled trial yet. We only considered the raw results of Vmax, $\triangle \mathrm{PD}$, and $\mathrm{LT}$, and therefore, did not take into consideration the pupillary reactivity indicator calculated by the Neurolight ${ }^{\circledR}$.

\section{Conclusions}

Vmax and $\triangle P D$ seem to be useful for the evaluation of the sedation in ICU compared with the BIS. This noninvasive technique of monitoring the depth of sedation can be highly beneficial especially for patients under myorelaxant drugs due to the impossibility of using any clinical sedation scales and the fact that these drugs do not alter PMR. This equipment could help 
reduce the risk of overdose or under dose of hypnotics in the ICU. A larger study is necessary to confirm these results and define cutoff values.

\section{Competing interests}

The authors declare that they have no competing interests.

\section{Authors' contributions}

OR Carried out the measurements and wrote the article. AWT Carried out all the statistical paragraph and corrected the article. QD Helped including patients in the study and corrected the article. LM Helped including patients in the study and corrected the article. TF Carried out the study plan and corrected the article. PR Carried out the study plan and corrected the article. DJ Carried out all the statistical part of the study and corrected the article. JC Suppervised the study, carried out the study plan and corrected the article. All authors read and approved the final manuscript.

\section{Author details}

'Service de Réanimation Polyvalente Hôpital Robert Debré, CHU 51092 Reims Cedex, France. ${ }^{2}$ Unité d'analyse méthodologique, CHU 51092 Reims Cedex, France.

Received: 29 August 2012 Accepted: 4 January 2013

Published: 22 February 2013

\section{References}

1. Payen JF, Chanques G, Mantz J, Hercule C, Auriant I, Leguillou JL, et al: Current practices in sedation and analgesia for mechanically ventilated critically ill patients: a prospective multicenter patient-based study. Anesthesiol 2007, 106(4):687-695. quiz 891-2.

2. Sessler CN, Grap MJ, Brophy GM: Multidisciplinary management of sedation and analgesia in critical care. Semin Respir Crit Care Med 2001, 22(2):211-226.

3. Park G, Coursin D, Ely EW, England M, Fraser GL, Mantz J, et al: Commentary, Balancing sedation and analgesia in the critically ill. Crit Care Clin 2001, 17(4):1015-1027.

4. French Society of Anesthesiology: Recommendations for sedation, analgesia and curarization. Short text. Societe Francaise d'Anesthesie et de Reanimation. Ann Fr Anesth Reanim 2000, 19(5):fig8-fi105.

5. Leslie K, Sessler DI, Smith WD, Larson MD, Ozaki M, Blanchard D, et al: Prediction of movement during propofol/nitrous oxide anesthesia. Performance of concentration, electroencephalographic, pupillary, and hemodynamic indicators. Anesthesiol 1996, 84(1):52-63.

6. Larson MD, Kurz A, Sessler DI, Dechert M, Bjorksten AR, Tayefeh F: Alfentanil blocks reflex pupillary dilation in response to noxious stimulation but does not diminish the light reflex. Anesthesiol 1997, 87(4):849-855.

7. Belani KG, Sessler DI, Larson MD, Lopez MA, Washington DE, Ozaki M, et al: The pupillary light reflex, Effects of anesthetics and hyperthermia. Anesthesiol 1993, 79(1):23-27.

8. Knaggs RD, Crighton IM, Cobby TF, Fletcher AJ, Hobbs GJ: The pupillary effects of intravenous morphine, codeine, and tramadol in volunteers. Anesth Analg 2004, 99(1):108-112.

9. Murray RB, Adler MW, Korczyn AD: The pupillary effects of opioids. Life Sci 1983, 33(6):495-509.

10. Larson MD: Mechanism of opioid-induced pupillary effects. Clin Neurophysiol 2008, 119(6):1358-1364.

11. Vivien B, Di Maria S, Ouattara A, Langeron O, Coriat P, Riou B: Overestimation of Bispectral index in sedated intensive care unit patients revealed by administration of muscle relaxant. Anesthesiol 2003, 99(1):9-17.

12. Sessler CN, Gosnell MS, Grap MJ, Brophy GM, O'Neal PV, Keane KA, et al: The Richmond Agitation-sedation scale: validity and reliability in adult intensive care unit patients. Am J Respir Crit Care Med 2002, 166(10):1338-1344.

13. Chanques G, Jaber S, Barbotte E, Verdier R, Henriette K, Lefrant JY, et al: Validation of the french translated Richmond vigilance-agitation scale. Ann Fr Anesth Reanim 2006, 25(7):696-701.

14. Ely EW, Truman B, Shintani A, Thomason JW, Wheeler AP, Gordon S, et al: Monitoring sedation status over time in ICU patients: reliability and validity of the Richmond Agitation-Sedation Scale (RASS). JAMA 2003, 289(22):2983-2991.
15. Larson MD, Talke PO: Effect of dexmedetomidine, an alpha2adrenoceptor agonist, on human pupillary reflexes during general anaesthesia. Br J Clin Pharmacol 2001, 51(1):27-33.

16. Eilers $\mathrm{H}$, Larson MD: The effect of ketamine and nitrous oxide on the human pupillary light reflex during general anesthesia. Auton Neurosci 2010, 152(1-2):108-114.

17. Johansen JW, Sebel PS: Development and clinical application of electroencephalographic bispectrum monitoring. Anesthesiol 2000, 93(5):1336-1344.

18. Dereu D, Lukaszewicz AC, Bart F: Prédiction par la pupillométrie du besoin d'anagésie lors de pansements chirurgicaux chez les patients sédatés de réanimation, SFAR. 2010.

19. Delsuc C, Calderon, Portran P: Pupillométrie et anesthésie, SFAR. 2010

20. Gray AT, Krejci ST, Larson MD: Neuromuscular blocking drugs do not alter the pupillary light reflex of anesthetized humans. Arch Neurol 1997, 54(5):579-584.

21. Thuong M: Sedation and analgesia assessment tools in ICU patients. Ann Fr Anesth Reanim 2008, 27(7-8):581-595.

22. Turkmen A, Altan A, Turgut N, Vatansever S, Gokkaya S: The correlation between the Richmond agitation-sedation scale and bispectral index during dexmedetomidine sedation. Eur J Anaesthesiol 2006, 23(4):300-304.

23. Brocas E, Dupont H, Paugam-Burtz C, Servin F, Mantz J, Desmonts JM: Bispectral index variations during tracheal suction in mechanically ventilated critically ill patients: effect of an alfentanil bolus. Intensive Care Med 2002, 28(2):211-213.

24. Deogaonkar A, Gupta R, DeGeorgia M, Sabharwal V, Gopakumaran B, Schubert $A$, et al: Bispectral Index monitoring correlates with sedation scales in brain-injured patients. Crit Care Med 2004, 32(12):2403-2406.

25. Consales G, Chelazzi C, Rinaldi S, De Gaudio AR: Bispectral index compared to Ramsay score for sedation monitoring in intensive care units. Minerva Anestesiol 2006, 72(5):329-336.

26. Frenzel D, Greim CA, Sommer C, Bauerle K, Roewer N: Is the bispectral index appropriate for monitoring the sedation level of mechanically ventilated surgical ICU patients? Intensive Care Med 2002, 28(2):178-183.

27. Nasraway SS Jr, Wu EC, Kelleher RM, Yasuda CM, Donnelly AM: How reliable is the Bispectral Index in critically ill patients? a prospective, comparative, single-blinded observer study. Crit Care Med 2002, 30(7):1483-1487.

28. Riess ML, Graefe UA, Goeters C, Van Aken H, Bone HG: Sedation assessment in critically ill patients with bispectral index. Eur J Anaesthesiol 2002, 19(1):18-22.

\section{doi:10.1186/2110-5820-3-5}

Cite this article as: Rouche et al.: Evaluation of the depth of sedation in an intensive care unit based on the photo motor reflex variations measured by video pupillometry. Annals of Intensive Care 2013 3:5.

\section{Submit your manuscript to a SpringerOpen ${ }^{\odot}$ journal and benefit from:}

- Convenient online submission

- Rigorous peer review

- Immediate publication on acceptance

- Open access: articles freely available online

- High visibility within the field

- Retaining the copyright to your article

Submit your next manuscript at $>$ springeropen.com 